\title{
Impact of Bevacizumab on parenchymal damage and functional recovery of the liver in patients with colorectal liver metastases
}

\author{
Andreas M. Volk ${ }^{\dagger}$, Johannes Fritzmann ${ }^{\dagger}$, Christoph Reissfelder, Georg F. Weber, Jürgen Weitz and Nuh N. Rahbari
}

\begin{abstract}
Background: Little is known about the safety of the anti-VEGF antibody bevacizumab in patients undergoing resection for colorectal liver metastases (CLM). This meta-analysis evaluates the impact of bevacizumab on parenchymal damage and functional recovery in patients undergoing resection for CLM.

Methods: The Medline, Embase and Cochrane Library were systematically searched for studies on preoperative chemotherapy with and without bevacizumab prior to resection of CLM. Studies that reported histological and/or clinical outcomes were eligible for inclusion. Meta-analyses were performed using a random effects model.

Results: A total of 18 studies with a total sample size of 2430 patients (1050 patients with bevacizumab) were found. Meta-analyses showed a significant reduction in sinusoidal obstruction syndrome (SOS) (Odds ratio 0.50 [95\% confidence interval $\left.0.37,0.67] ; p<0.001 ;\left.\right|^{2}=0 \%\right)$ and hepatic fibrosis $\left(0.61[0.4,0.86] ; p=0.004 ;\left.\right|^{2}=7 \%\right)$ after preoperative chemotherapy with bevacizumab. The reduced incidence of posthepatectomy liver failure in patients with bevacizumab treatment just failed to reach statistical significance $\left(0.61[0.34,1.07] ; p=0.08 I^{2}=6 \%\right)$. While there was no difference in perioperative morbidity and mortality, the incidence of wound complications was significantly increased in patients who received bevacizumab $\left(1.81[1.12,2.91] ; p=0.02 \mathrm{I}^{2}=4 \%\right)$.

Conclusions: The combination of bevacizumab with cytotoxic chemotherapy is safe but increases the incidence of wound complications after resection of CLM. The reduction of SOS and hepatic fibrosis warrant further investigation and may explain the inverse association of bevacizumab administration and posthepatectomy liver failure.
\end{abstract}

Keywords: Bevacizumab, Chemotherapy, Liver resection, Parenchymal damage, Complications

\section{Background}

Complete surgical resection remains the only curative option in patients with colorectal liver metastases (CLM) enabling 5-year overall survival rates of $50 \%[1,2]$. Effective oxaliplatin- and irinotecan-based chemotherapy protocols together with targeted agents have significantly improved objective response rates, conversion to resectability and long-term survival in metastatic colorectal cancer not amenable to curative resection [3-6]. As a consequence of

\footnotetext{
* Correspondence: nuh.rahbari@uniklinikum-dresden.de

${ }^{\dagger}$ Equal contributors

Department of Visceral, Thoracic and Vascular Surgery, University Hospital Carl Gustav Carus, Technical University Dresden, Fetscherstr. 74, D-01307

Dresden, Germany
}

the increased use of modern combination chemotherapy protocols, a growing number of patients undergo hepatic resection after treatment with cytotoxic and molecular targeted agents. Hepatic toxicity of irinotecan and oxaliplatin-containing regimens are well-described and typically manifest as chemotherapy-associated steatohepatitis (CASH) and sinusoidal obstruction syndrome (SOS), respectively. However, much less is known about the effects of targeted agents on parenchymal damage to the liver and their influence on perioperative outcome after hepatic resection. Among targeted agents approved for treatment of metastatic colorectal cancer, the impact of bevacizumab, a monoclonal antibody against the vascular endothelial growth factor A (VEGF-A) on liver histology

\section{$\int$ Biomed Central}


and perioperative complications is of particular interest. Besides its role in pathological angiogenesis, the VEGF family of growth factors exerts important physiological functions. The important function of VEGF in homeostasis of the liver microenvironment, liver regeneration and wound healing have therefore raised concerns about the safety of bevacizumab in the peri-operative setting of patients undergoing hepatic resection. To date, several reports have been published on the effects of bevacizumab on liver-parenchymal damage, functional recovery and perioperative outcome after resection of CLM with in part conflicting results [7-10].

The aim of this systematic review and meta-analysis was to evaluate the effects of preoperative bevacizumab administration on histological and perioperative outcomes of patients undergoing surgical resection of CLM.

\section{Methods}

This systematic review and meta-analysis was conducted in accordance to the PRISMA statement [11].

\section{Search strategy and selection criteria}

A computerized search of the Medline, Embase and Cochrane Library databases was performed in May 2014 using the following search terms in various combinations: 'Colon,' 'Rectal,' 'Colorectal,' 'Liver,' 'Hepatic', 'Metastases', 'Bevacizumab, 'Avastin'. To find other potentially eligible studies, the reference lists of relevant articles were searched manually. First, the search findings were screened for potentially eligible studies based on the titles and abstracts. For references that were considered potentially relevant, the full articles were obtained for detailed evaluation using the following selection criteria: All studies (prospective or retrospective) that reported the impact of preoperative bevacizumab administration on perioperative outcome and/or liver histology of patients undergoing resection of CLM were eligible for inclusion. For studies to be eligible for inclusion, at least one predefined outcome for patients treated with chemotherapy with and without addition of bevacizumab had to be reported within one study/report. Comments and letters were excluded as were studies that were not published in a peer-reviewed journal. Furthermore, studies that were published in a language other than English, German or French were excluded. In case of multiple publications from the same institution with identical or overlapping patient cohorts the most informative report was included.

\section{Data extraction and quality assessment}

Two authors (N.N.R. and A.M.V.) independently extracted the following data from each identified study: first author, year of publication, study period, study, design, sample size, baseline characteristics of the study cohort, kind of concomitant chemotherapy, number of preoperative chemotherapy cycles with Bevacizumab, time interval between last Bevacizumab administration and surgery. The following histological parameters were recorded separately for patients with and without preoperative administration of Bevacizumab: sinusoidal obstruction syndrome (total and moderate/severe), hepatic fibrosis, hepatic steatosis, complete pathological response and complete (R0) tumor resection. With regard to perioperative outcomes data on the following endpoints were documented: perioperative morbidity and mortality, wound complications, liver failure. Disagreements were resolved by discussion.

To assess the methodological quality of included studies, the risk of bias tool recommended by the Cochrane Collaboration was applied [12]. The criteria proposed by the Grading of Recommendations, Assessment, Development and Evaluation (GRADE) Working Group (www.gradeworkinggroup.org) were used for evaluation of non-randomized studies [13-15]. The following criteria were evaluated for each included study: application of adequate eligibility criteria, adequate measurement of outcomes, adequate control of confounding factors, completeness of follow-up and adequacy of its duration, adequate reporting of outcomes and absence of other sources of bias. The use of scales with scores for multiple items that are summed up is discouraged by the Cochrane Collaboration. The above criteria were therefore used to grade individual studies as high or low risk of bias $[12,15,16]$.

\section{Statistical analyses}

Meta-analyses were performed for outcomes for which at least two of the included studies provided comparative data for patients who underwent liver resection after preoperative chemotherapy with and without Bevacizumab. Odds ratio (OR) was chosen as effect measure dichotomous data, which was reported together with the $95 \%$ confidence interval (CI). Meta-analyses were carried out using a random effects model for more conservative effect estimates due to potential inter-study heterogeneity regarding study populations, chemotherapy protocols and definitions of outcome parameters [17]. Heterogeneity was assessed with $\mathrm{I}^{2}$ statistics. This approach describes the proportion of total variation observed between the trials that is attributable to differences between trials rather than sampling error (chance) [18]. Moderate to high degree of statistical heterogeneity was assumed in case of an $\mathrm{I}^{2}$ value of more than $30 \%$. Reasons for statistical heterogeneity were explored using sensitivity analyses (exclusion of individual studies). Furthermore, subgroup analyses carried out to evaluate the impact treatment duration, time interval between last bevacizumab treatment and surgery and kind of concomitant chemotherapy on the results. 
Presence of publication bias was evaluated using Funnel plot analyses [19].

Meta-analyses were carried out using Review Manager Version 5.0 software (Copenhagen: The Nordic Cochrane Centre; The Cochrane Collaboration, 2008).

\section{Results}

The systematic literature search identified 18 relevant studies (Fig. 1) [7, 8, 10, 20-34]. These studies had a cumulative sample size of 2430 patients, of which 1050 patients received bevacizumab prior to resection of CLM (Table 1). The included studies were published between 2007 and 2014. More than six cycles of preoperative treatment with bevacizumab was administered in five studies $[7,20,22,26,33]$, whereas in the remaining studies six or less cycles of chemotherapy with bevacizumab was given. The average time interval between the last dose of bevacizumab and the date of surgery was eight weeks or less in seven studies (Fig.2) $[8,22,24,25,30,33,34]$ and more than eight weeks in eight studies [7, 10, 20, 21, 23, 26, 27, 31]. Bevacizumab was combined with oxaliplatin-based chemotherapy regimen in the majority $(>76 \%)$ of study patients in eight of the included studies [8, 24, 27-31, 34].

\section{Histological analyses}

A total of seven studies with 1206 patients provided data on SOS (Additional file 1: Table S1) [8, 10, 24, 26, 28, 29, 31]. Meta-analysis of the results from these studies showed a statistically significant reduction in SOS for patients who received chemotherapy with bevacizumab with no statistical heterogeneity $(0.50 \quad[0.37$, 0.67]; $\left.p<0.001 ; \mathrm{I}^{2}=0 \%\right)$. This association was confirmed for the development of moderate and severe SOS $\left(0.31[0.18,0.53] ; p<0.001 ; \mathrm{I}^{2}=37 \%\right)$, which was reported in seven studies $[8,10,20,24,26,29,31]$. Sensitivity analyses revealed that heterogeneity was caused by the study of Aussilhou et al. [20]. Exclusion of this study completely removed statistical heterogeneity $(0.25[0.17$, 0.38 ]; $p<0.001 ; \mathrm{I}^{2}=0 \%$ ). Subgroup analyses confirmed the

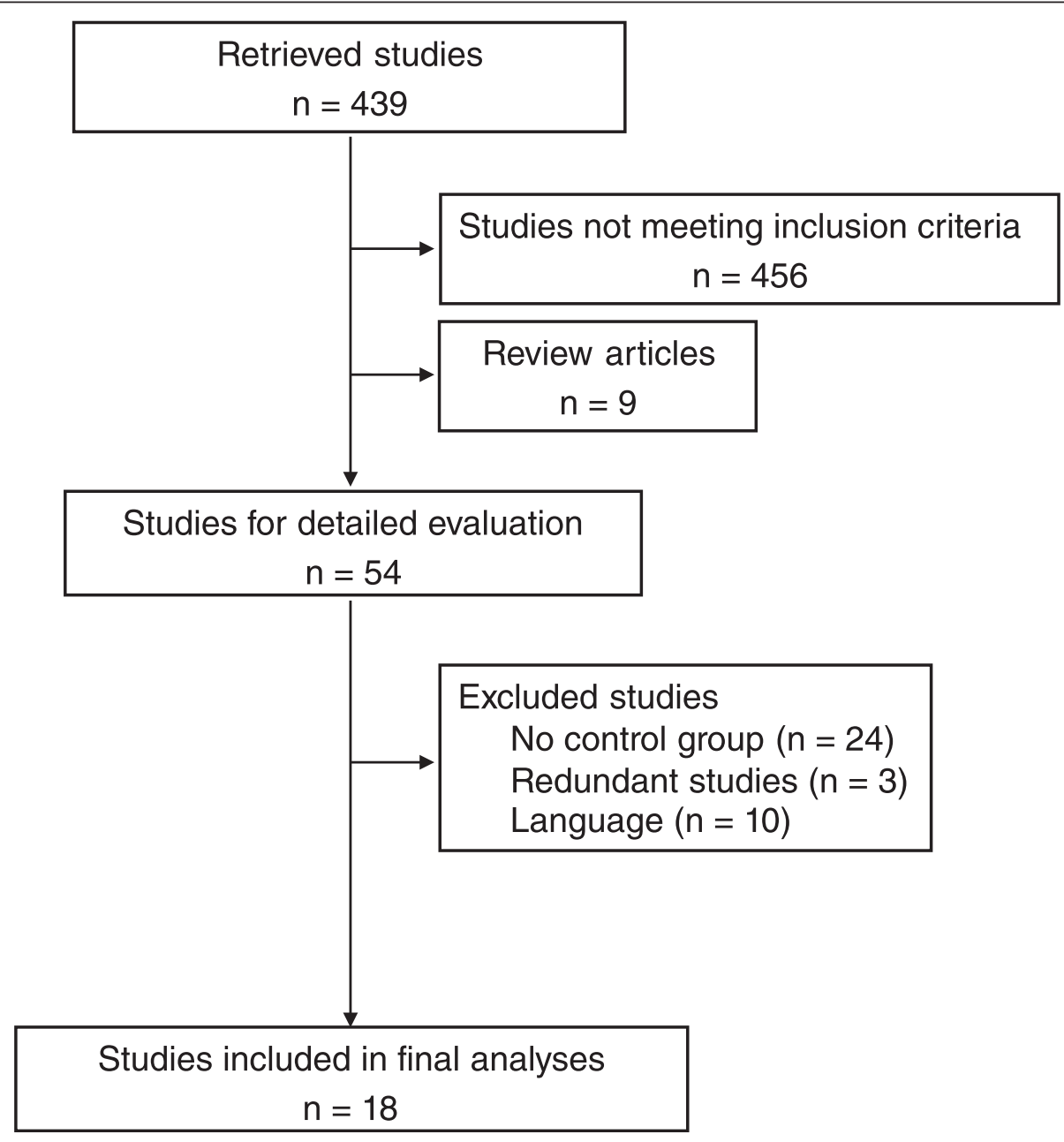

Fig. 1 Flow chart of study selection 
Table 1 Characteristics of identified studies

\begin{tabular}{|c|c|c|c|c|c|c|c|c|}
\hline Reference & Year & Inclusion period & $\begin{array}{l}\text { Sample size } \\
\text { (total/BEV) }\end{array}$ & Study type & $\begin{array}{l}\text { CTX in BEV group } \\
\mathrm{OX} / \mathrm{IRI} / \mathrm{OX}+\mathrm{IRI}[\%]\end{array}$ & No BEV cycles & $\begin{array}{l}\text { Interval last BEV cycle } \\
\text { to surgery }\end{array}$ & Risk of bias \\
\hline Aussilhou & 2009 & $2002-2008$ & $40 / 13$ & Prosp. CS & $54 / 38$ & $12(2-36)$ & 92 days & High \\
\hline Constantinidou & 2013 & Until 9/2010 & $94 / 42$ & Retrosp. CS & $64 / 33$ & $4.5(4-12)^{a}$ & 73 (44-141) days & High \\
\hline D’Angelica & 2007 & 2004-2005 & $64 / 32$ & Matched CCS & $56 / 37$ & $9(4-15)$ & 6.9 (3-15) weeks & High \\
\hline Kesmodel & 2008 & 2004-2006 & $125 / 81$ & Retrosp. CS & $70 / 36$ & $84(14-513)^{b}$ & 58 (31-117) days & High \\
\hline Klinger & 2009 & 2001-2006 & $106 / 56$ & Retrosp. $\mathrm{CS}^{\mathrm{e}}$ & $100 / 0$ & 5 & 5 weeks & High \\
\hline Lubezky & 2013 & 2000-2007 & $191 / 134$ & Retrosp. CS & $72 / 28$ & - & $>6$ weeks & High \\
\hline Mahfud & 2010 & 2005-2007 & $90 / 45$ & Matched CCS & $24 / 71$ & $9(7-10)^{a}$ & 9 weeks $(60,47-73$ days) & Low \\
\hline Millet & 2012 & 2006-2011 & $82 / 41$ & Matched CCS & $15 / 85$ & $6(4-16)^{a}$ & 65 (39-90) days & Low \\
\hline Pessaux & 2010 & 2005-2007 & $42 / 21$ & Matched CCS & $76 / 10 / 14$ & $8.1 \pm 4.7$ & $11.7 \pm 4.7$ weeks & Low \\
\hline Reddy & 2008 & 1996-2006 & $96 / 39$ & Retrosp. CS & $79 / 21$ & $6(3-8)^{c}$ & 10 (8-13) weeks & High \\
\hline Ribero & 2007 & $2002-2006$ & $105 / 62$ & Retrosp. CS & $100 / 0$ & $6(3-12)^{a}$ & $>6$ weeks & High \\
\hline Rong & 2014 & 2002-2012 & $501 / 117$ & Retrosp. CS & $100 / 0$ & $6 \pm 12^{a}$ & - & High \\
\hline Rubbia Brandt & 2010 & - & $274 / 70^{d}$ & Retrosp. CS & $100 / 0$ & - & - & High \\
\hline Tamandl & 2009 & 2005-2009 & $214 / 102$ & Retrosp. CS & $82 / 13$ & $6(1-20)$ & 34 (17-99) days & High \\
\hline van der Pool & 2012 & 2003-2008 & $104 / 51$ & Retrosp. CS & $100 / 0$ & $4(1-15)^{a}$ & 11 (5-38) weeks & High \\
\hline Vera & 2014 & 2005-2011 & $95 / 51$ & Retrosp. CS & $45 / 53$ & $6(1-21)^{a}$ & - & High \\
\hline Wicherts & 2011 & 2005-2009 & $164 / 67$ & Retrosp. CS & $23 / 68 / 2$ & $8.6(1-34)$ & 8 (3-19) weeks & Low \\
\hline Zorzi & 2008 & $1995-2007$ & $43 / 26$ & Retrosp. CS & $100 / 0$ & $5(3-20)^{a}$ & 7.9 (3-36) weeks & High \\
\hline
\end{tabular}

${ }^{a}$ Number of CTx cycles

${ }^{\mathrm{b}}$ Duration of BEV treatment in days

'Duration of BEV treatment in months

dIrinotecan was added in 79 patients of the whole study cohort

${ }^{e}$ Combined retrospective analysis of two phase II trials. Continuous data are presented as median (range) or mean (standard deviation) based on the kind of data presented in the original publication

protective effect of preoperative chemotherapy combined with bevacizumab on total as well as moderate/severe SOS throughout all evaluated strata (Table 2).

The definition of significant fibrosis applied in the identified studies is summarized in Additional file 2: Table S2. Meta-analysis showed a significant reduction of hepatic fibrosis in patients who received preoperative chemotherapy with bevacizumab before resection of $\operatorname{CLM}\left(0.61[0.4,0.86] ; p=0.004 ; \mathrm{I}^{2}=7 \%\right)$. Subgroup analyses suggested the reduction of hepatic fibrosis to be more pronounced after $\leq 6$ cycles of bevacizumab $(0.60$ $\left.[0.38,0.94] ; p=0.03 ; \mathrm{I}^{2}=0 \%\right)$ compared to $>6$ cycles of bevacizumab $\left(0.70[0.30,1.63] ; p=0.41 ; \mathrm{I}^{2}=36 \%\right)$ and in case a high proportion of patients received an oxaliplatinbased chemotherapy regimen $(0.52[0.37,0.75] ; p<0.001$; $\left.\mathrm{I}^{2}=0 \%\right)$ compared to a lower fraction of patients with an oxaliplatin-based chemotherapy regimen $(0.70[0.30,1.63]$; $\left.p=0.41 ; \mathrm{I}^{2}=36 \%\right)$. In these analyses statistical heterogeneity was caused by the study by Wicherts et al. [33]. Exclusion of this study completely resolved statistical heterogeneity $\left(0.43[0.19,1.0] ; p=0.05 ; \mathrm{I}^{2}=0 \%\right)$ in the subgroups of patients with $>6$ cycles of bevacizumab and a lower fraction of oxaliplatin-based chemotherapy regimens.

In total, seven studies with a sample size of 1116 patients provided results on hepatic steatosis after preoperative chemotherapy with and without bevacizumab [10, 20, 26, 28, 29, 31, 33]. Pooled analysis of the results from these studies indicated no impact of preoperative chemotherapy with bevacizumab on hepatic steatosis $\left(0.96[0.63,1.45] ; p=0.83 ; \mathrm{I}^{2}=30 \%\right)$. The lack of association between preoperative bevacizumab administration and hepatic steatosis was confirmed throughout the performed subgroup analyses.

In further analyses the effect of preoperative chemotherapy with and without bevacizumab on complete (R0) resection of liver metastases and complete pathologic response was evaluated. These analyses revealed no significant association between preoperative bevacizumab treatment and R0 (0.71 $\left.[0.32,1.59] ; p=0.40 ; \mathrm{I}^{2}=58 \%\right)$ and complete pathologic response $\left(1.51[0.83,2.75] ; p=0.18 ; \mathrm{I}^{2}=9 \%\right)$.

\section{Functional recovery and perioperative outcome}

Perioperative outcomes are summarized in Table 3 . Meta-analysis showed no statistically significant difference in perioperative morbidity between patients with and without preoperative bevacizumab treatment with low statistical heterogeneity $(1.10[0.88,1.37] ; p=0.39$; $\left.\mathrm{I}^{2}=10 \%\right)$. However, subgroup analyses revealed increased perioperative complications in patients who received preoperative chemotherapy with bevacizumab for 
Table 2 Meta-analyses on outcomes of parenchymal damage and perioperative outcomes after preoperative chemotherapy with and without bevacizumab for CLM

\begin{tabular}{|c|c|c|c|c|c|}
\hline \multicolumn{6}{|l|}{ Histological analyses } \\
\hline & Subgroup & SOS & Moderate/Severe SOS & Hepatic fibrosis & Hepatic steatosis \\
\hline \multirow[t]{2}{*}{ Total } & - & $0.50[0.37,0.67]$ & $0.25[0.17,0.38]$ & $0.61[0.44,0.86]$ & $0.96[0.63,1.45]$ \\
\hline & & $p<0.001 ;\left.\right|^{2}=0 \%$ & $p<0.001 ; 1^{2}=0 \%$ & $p=0.004 ; 1^{2}=7 \%$ & $p=0.83 ;\left.\right|^{2}=30 \%$ \\
\hline \multirow[t]{4}{*}{ No of BEV cycles } & $\leq 6$ & $0.50[0.34,0.72]$ & $0.21[0.11,0.41]$ & $0.60[0.38,0.94]$ & $0.94[0.56,1.59]$ \\
\hline & & $p<0.001 ;\left.\right|^{2}=0 \%$ & $p<0.001 ; 1^{2}=0 \%$ & $p=0.03 ;\left.\right|^{2}=0 \%$ & $p=0.82 ;\left.\right|^{2}=53 \%$ \\
\hline & $>6$ & $0.46[0.21,1.01]$ & $0.29[0.10,0.81]$ & $0.43[0.19,1.0]$ & $0.93[0.34,2.52]$ \\
\hline & & $p=0.05 ;\left.\right|^{2}=0 \%$ & $p=0.02 ; i^{2}=0 \%^{\mathrm{a}}$ & $p=0.05 ; 1^{2}=0 \%{ }^{b}$ & $p=0.89 ;\left.\right|^{2}=31 \%$ \\
\hline \multirow[t]{4}{*}{ Time last BEV cycle to surgery } & $\leq 8$ weeks & $0.47[0.23,0.95]$ & $0.21[0.10,0.47]$ & - & - \\
\hline & & $p=0.04 ; I^{2}=32 \%$ & $p<0.001 ; 1^{2}=0 \%$ & & \\
\hline & $>8$ weeks & $0.50[0.28,0.86]$ & $0.26[0.12,0.56]$ & $0.61[0.34,1.09]$ & $0.63[0.21,1.84]$ \\
\hline & & $p=0.01 ;\left.\right|^{2}=0 \%$ & $p<0.001 ; 1^{2}=0 \%$ & $p=0.09 ;\left.\right|^{2}=0 \%$ & $p=0.39 ;\left.\right|^{2}=45 \%$ \\
\hline \multirow[t]{4}{*}{ Cytotoxic chemotherapy ${ }^{4}$} & Oxaliplatin high & $0.46[0.21,1.01]$ & $0.29[0.10,0.81]$ & $0.43[0.19,1.00]$ & $0.93[0.34,2.52]$ \\
\hline & & $p=0.05 ;\left.\right|^{2}=0 \%$ & $p=0.02 ;\left.\right|^{2}=0 \%^{\mathrm{a}}$ & $p=0.05 ; 1^{2}=0 \%{ }^{b}$ & $p=0.89 ;\left.\right|^{2}=31 \%$ \\
\hline & Oxaliplatin low & $0.51[0.37,0.69]$ & $0.25[0.16,0.38]$ & $0.52[0.37,0.75]$ & $0.94[0.56,1.59]$ \\
\hline & & $p<0.001 ; 1^{2}=0 \%$ & $p<0.001 ; 1^{2}=0 \%$ & $p<0.001 ;\left.\right|^{2}=0 \%$ & $p=0.82 ;\left.\right|^{2}=53 \%$ \\
\hline \multicolumn{6}{|c|}{ Functional Recovery and Perioperative Outcome } \\
\hline & & Morbidity & Wound complications & Liver failure & Mortality \\
\hline \multirow[t]{2}{*}{ Total } & - & $1.10[0.88,1.37]$ & $1.81[1.12,2.91]$ & $0.61[0.34,1.07]$ & $0.60[0.20,1.82]$ \\
\hline & & $p=0.39 ; 12=10 \%$ & $p=0.02 ; 12=4 \%$ & $p=0.08 ; 12=6 \%$ & $p=0.37 ; 12=0 \%$ \\
\hline \multirow[t]{4}{*}{ No of BEV cycles } & $\leq 6$ & $0.99[0.75,1.30]$ & $1.22[0.61,2.42]$ & $0.49[0.21,1.14]$ & $0.54[0.10,2.93]$ \\
\hline & & $p=0.95 ; 12=0 \%$ & $p=0.58 ; 12=0 \%^{c}$ & $p=0.10 ; 12=0 \%$ & $p=0.47 ; 12=0 \%$ \\
\hline & $>6$ & $1.51[1.08,2.13]$ & $1.88[0.89,3.99]$ & $0.85[0.36,2.00]$ & $0.46[0.09,2.41]$ \\
\hline & & $p=0.02 ; 12=0 \%$ & $p=0.10 ; 12=0 \%$ & $p=0.71 ; 12=16 \%$ & $p=0.36 ; 12=0 \%$ \\
\hline \multirow[t]{4}{*}{ Time last BEV cycle to surgery } & $\leq 8$ weeks & $1.06[0.70,1.59]$ & $1.59[0.50,5.11]$ & $0.50[0.16,1.57]$ & $1.16[0.14,9.37]$ \\
\hline & & $p=0.80 ; 12=33 \%$ & $p=0.43 ; 12=0 \%$ & $p=0.24 ; 12=0 \%$ & $p=0.89 ; 12=0 \%$ \\
\hline & $>8$ weeks & $1.27[0.92,1.74]$ & $1.45[0.84,2.50]$ & $0.70[0.32,1.51]$ & $0.47[0.13,1.71]$ \\
\hline & & $p=0.15 ; 12=0 \%$ & $p=0.19 ; 12=0 \%$ & $p=0.36 ; 12=23 \%$ & $p=0.25 ; 12=0 \%$ \\
\hline \multirow[t]{4}{*}{ Cytotoxic chemotherapy $^{d}$} & Oxaliplatin high & $1.19[0.87,1.63]$ & $1.47[0.87,2.49]$ & $0.61[0.28,1.30]$ & $0.55[0.14,2.19]$ \\
\hline & & $p=0.28 ; 12=17 \%$ & $p=0.15 ; 12=0 \%$ & $p=0.20 ; 12=29 \%$ & $p=0.40 ; 12=0 \%$ \\
\hline & Oxaliplatin low & $1.01[0.74,1.38]$ & $3.19[0.82,12.35]$ & $0.50[0.16,1.59]$ & $0.70[0.11,4.47]$ \\
\hline & & $p=0.95 ; 12=6 \%$ & $p=0.09 ; 12=51 \%$ & $p=0.24 ; 12=0 \%$ & $p=0.71 ; 12=0 \%$ \\
\hline
\end{tabular}

${ }^{a}$ Results of sensitivity analyses after exclusion of the study by Aussilhou et al. [20]

${ }^{\mathrm{b}}$ Results of sensitivity analyses after exclusion of the study by Wicherts et al. [33]

'Results of sensitivity analyses after exclusion of the study by Rong et al. [28]

${ }^{\mathrm{d}}$ The subgroup analysis on the kind of systemic chemotherapy administered was based on the fraction of patients who received oxaliplatin or irinotecan. Studies in the oxaliplatin high group had $>76 \%$ of patients who received oxaliplatin, whereas studies in the oxaliplatin low had $\leq 76 \%$ patients with oxaliplatin. This cut-off was chosen based on the average proportion of patients with oxaliplatin in each study

more than six cycles $\left(1.51[1.08,2.13] ; p=0.02 ; \mathrm{I}^{2}=0 \%\right)$. Neither the duration between the last dose of bevacizumab and the date of surgery nor the kind of cytotoxic therapy that was combined with bevacizumab had a significant impact on perioperative morbidity. Metaanalysis just failed to show a significant impact of preoperative bevacizumab treatment on severe complications $\left(1.39[0.96,2.01] ; p=0.08 ; \mathrm{I}^{2}=0 \%\right)$.
Due to the role of VEGF-A in physiological wound healing, the influence of chemotherapy with bevacizumab on wound healing complications was analyzed. Meta-analyses revealed a significant association of preoperative bevacizumab administration and postoperative wound healing complications with no statistical heterogeneity $\left(1.81[1.12,2.91] ; p=0.02 ; \mathrm{I}^{2}=4 \%\right)$. Subgroup analyses indicated a more pronounced impact on wound 
Table 3 Perioperative outcomes reported in studies on preoperative chemotherapy with and without bevacizumab for CLM

\begin{tabular}{|c|c|c|c|c|c|c|c|c|c|c|c|}
\hline Reference & Group & $\begin{array}{l}\text { Duration of surgery } \\
\text { (min) }\end{array}$ & $\begin{array}{l}\text { Estimated blood loss } \\
(\mathrm{ml})\end{array}$ & $\begin{array}{l}\text { Morbidity } \\
(\%)\end{array}$ & $\begin{array}{l}\text { Severe morbidity } \\
(\%)\end{array}$ & $\begin{array}{l}\text { Wound compl. } \\
(\%)\end{array}$ & $\begin{array}{l}\text { Liver failure } \\
(\%)\end{array}$ & $\begin{array}{l}\text { Bile leakage } \\
(\%)\end{array}$ & $\begin{array}{l}\text { Thromboembolic } \\
\text { events (\%) }\end{array}$ & $\begin{array}{l}\text { Hospital stay } \\
\text { (d) }\end{array}$ & $\begin{array}{l}\text { Mortality } \\
(\%)\end{array}$ \\
\hline \multirow[t]{2}{*}{ Aussilhou } & BEV- & & & 50 & & & 15 & 5 & & & 5 \\
\hline & $\mathrm{BEV}+$ & & & 55 & & & 18 & 9 & & & 0 \\
\hline \multirow[t]{2}{*}{ Constantinidou } & BEV- & & $650(100-2200)$ & 54 & 13 & 12 & 13 & & 0 & $10(4-32)$ & 2 \\
\hline & $\mathrm{BEV}+$ & & $540(50-2000)$ & 48 & 12 & 14 & 10 & & 5 & $10(4-57)$ & 0 \\
\hline \multirow[t]{2}{*}{ D’Angelica } & BEV- & - & $500(200-5000)$ & 38 & 9 & 6 & & & & 7 (n.r.) & 0 \\
\hline & $\mathrm{BEV}+$ & $235(85-500)$ & $300(0-1500)$ & 41 & 6 & 19 & & & & & 0 \\
\hline \multirow[t]{2}{*}{ Kesmodel } & BEV- & 134 (69-408) & $200(50-1750)$ & 43 & & 25 & 7 & 2 & & & 2 \\
\hline & $\mathrm{BEV}+$ & $139(67-675)$ & $250(50-1950)$ & 49 & & 28 & 1 & 4 & & & 1 \\
\hline \multirow[t]{2}{*}{ Lubezky } & BEV- & & & 47 & 9 & 2 & 5 & 9 & 4 & & 0 \\
\hline & $\mathrm{BEV}+$ & & & 35 & 11 & 2 & 1 & 8 & 5 & & 2 \\
\hline \multirow[t]{2}{*}{ Mahfud } & BEV- & $270(237-302)$ & $658(407-908)$ & 40 & 18 & 9 & 20 & & 9 & $13(8-18)$ & 4 \\
\hline & $\mathrm{BEV}+$ & $248(224-270)$ & $523(399-646)$ & 56 & 31 & 11 & 7 & & 7 & $15(11-20)$ & 0 \\
\hline \multirow[t]{2}{*}{ Millet } & BEV- & $300(175-645)$ & $500(50-1700)$ & 34 & 10 & & 12 & & & $8(5-21)$ & 0 \\
\hline & $\mathrm{BEV}+$ & $300(156-540)$ & $500(150-1100)$ & 56 & 12 & & 22 & & & $9(6-22)$ & 0 \\
\hline \multirow[t]{2}{*}{ Pessaux } & BEV- & $277( \pm 55)$ & $548( \pm 233)$ & 19 & & 0 & 0 & 0 & & $13.7( \pm 4.2)$ & 0 \\
\hline & $\mathrm{BEV}+$ & $321( \pm 160)$ & $983( \pm 1476)$ & 29 & & 14 & 0 & 0 & & $12.7( \pm 6.3)$ & 0 \\
\hline \multirow[t]{2}{*}{ Reddy } & BEV- & & $600(350-800)$ & 39 & 25 & 7 & 26 & & 5 & $7(6-9)$ & 4 \\
\hline & $\mathrm{BEV}+$ & & $425(263-600)$ & 44 & 28 & 10 & 18 & & 3 & $8(7-10)$ & 3 \\
\hline \multirow[t]{2}{*}{ Rong } & BEV- & & & 29 & & 1 & 4 & 7 & 1 & $10( \pm 50)$ & \\
\hline & $\mathrm{BEV}+$ & & & 26 & & 6 & 1 & 3 & 0 & $9( \pm 35)$ & \\
\hline \multirow[t]{2}{*}{ Tamandl } & BEV- & & & 34 & 7 & & & & & $9(5-47)$ & 0 \\
\hline & $\mathrm{BEV}+$ & & & 44 & 11 & & & & & $8(4-77)$ & 0 \\
\hline \multirow[t]{2}{*}{ Van der Pool } & BEV- & & & 32 & 6 & & & 4 & & $7(3-23)$ & 0 \\
\hline & $\mathrm{BEV}+$ & & & 25 & 8 & & & 2 & & $6(3-54)$ & 0 \\
\hline \multirow[t]{2}{*}{ Wicherts } & BEV- & $341( \pm 110)$ & & 36 & 5 & 14 & 2 & 3 & 1 & $11.3( \pm 7.1)$ & 0 \\
\hline & $\mathrm{BEV}+$ & $414( \pm 146)$ & & 43 & 16 & 8 & 2 & 6 & 2 & $13.6( \pm 14.4)$ & 0 \\
\hline \multirow[t]{2}{*}{ Zorzi } & BEV- & & & 54 & 39 & & 31 & & & & 8 \\
\hline & BEV+ & & & 42 & 16 & & 26 & & & & 5 \\
\hline
\end{tabular}




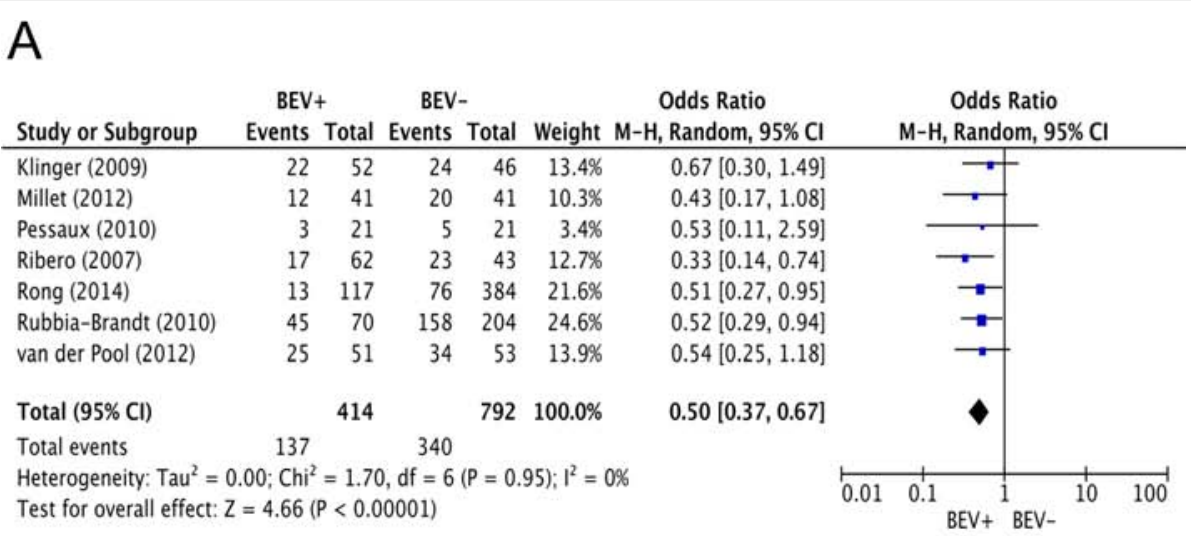

B

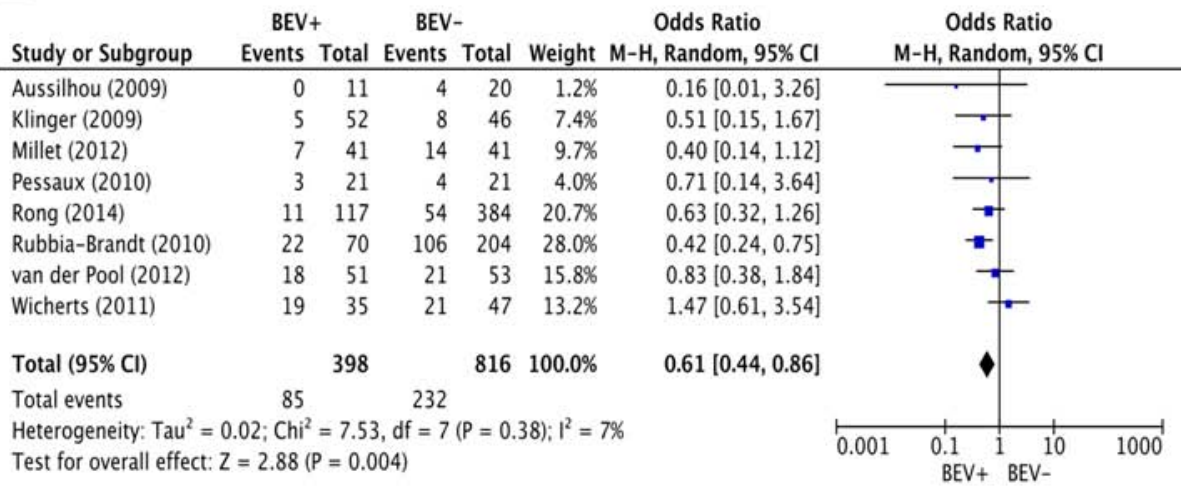

C

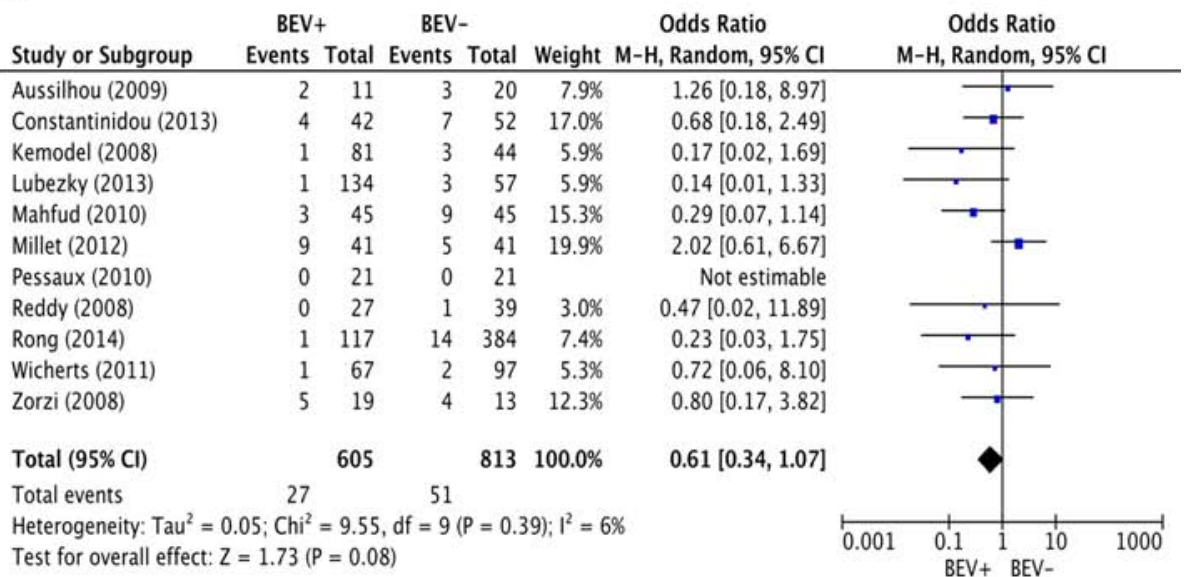

Fig. 2 Meta-analyses on the association of preoperative bevacizumab treatment with parenchymal damage and posthepatectomy liver failure in patients with CLM. a Association of bevacizumab treatment with sinusoidal obstruction syndrome (SOS). $\mathbf{b}$ Association of bevacizumab treatment with hepatic fibrosis. c Association of bevacizumab treatment with posthepatectomy liver failure

healing, if $>6$ cycles of bevacizumab were administered preoperatively.

Further, the effect of preoperative bevacizumab administration on postoperative liver dysfunction was analyzed. This analysis revealed a trend towards a decreased incidence of posthepatectomy liver failure in patients who received chemotherapy together with bevacizumab preoperatively $\left(0.61[0.34,1.07] ; p=0.08 \mathrm{I}^{2}=6 \%\right)$. Subgroup analyses failed to demonstrate an influence of the number of bevacizumab cycles and the time interval until surgery on the development of posthepatectomy liver failure. The reduction of this complication appeared to 
be more pronounced in studies with higher proportion of patients with irinotecan-based chemotherapy (0.61 $\left.[0.28,1.30] ; p=0.20 ; \mathrm{I}^{2}=29 \%\right)$. Sensitivity analyses revealed statistical heterogeneity in this subgroup of being caused by the study by Millet et al. [10] Removal of this study completely resolved statistical heterogeneity and indicated a statistically significant reduction of liver failure in the bevacizumab group $(0.44[0.21,0.89] ; p=0.02$; $\left.\mathrm{I}^{2}=0 \%\right)$. In total, 13 studies with a cumulative sample size of 1329 patients provided data on perioperative mortality [7, 10, 20-23, 25-27, 30, 31, 33, 34]. The pooled analysis of the results from these studies did not show a significant impact of chemotherapy combined with vs. without bevacizumab on perioperative mortality after liver resection $\left(0.60[0.20,1.82] ; p=0.37 ; \mathrm{I}^{2}=0 \%\right)$.

\section{Discussion}

It has been demonstrated previously that the chemotherapeutic agent oxaliplatin increases the risk of SOS [35]. This study shows that preoperative administration of bevacizumab is associated with a strong reduction of total SOS incidence as well as the incidence of moderate and severe SOS. Remarkably, subgroup analyses revealed that the reduced incidence of SOS was more pronounced in case $\leq 6$ cycles of chemotherapy with bevacizumab were administered suggesting that the protective effect tapers off with time. The mechanisms by which anti-VEGF therapy reduces the development of SOS remain incompletely understood. Besides biologic processes related to oxidative stress, remodeling of the extracellular matrix and the coagulation cascade, gene expression analyses have suggested angiogenic pathways to be involved in the pathogenesis of SOS [36, 37].

It is an interesting finding of the present study that preoperative treatment with bevacizumab significantly reduced hepatic fibrosis but had no impact on hepatic steatosis. This effect appeared to be more pronounced for a shorter period of preoperative chemotherapy and in case anti-VEGF therapy was given together with oxaliplatin-based chemotherapy. Previous studies have already demonstrated an anti-fibrotic activity of antiangiogenic agents in the hepatic parenchyma [38, 39]. Using a rat liver fibrosis model Wang et al. showed that sorafenib, a multiple receptor tyrosine kinase inhibitor that among others targets the VEGF receptor family (VEGFR-2 and VEGFR-3) and platelet-derived growth factor receptor family (PDGFR-beta and Kit) [40], reduces intrahepatic fibrogenesis. The anti-fibrotic effect of sorafenib may be mediated by targeting PDGFR which have been shown to play an important role in liver fibrogenesis [41]. Much less is known about the effects of the anti-VEGF antibody bevacizumab on remodeling of the extracellular matrix within the hepatic parenchyma. The results of the present study should therefore prompt further investigations elucidating the molecular mechnisms by which anti-VEGF therapy attenuated hepatic fibrosis in patients receiving systemic chemotherapy and, moreover, explore its potential as an anti-fibrotic agent in patients with liver fibrosis due to various etiologies.

Among other cytokines VEGF has been repeatedly shown to be involved in the process of liver regeneration $[42,43]$. Introduction of anti-VEGF agents have therefore raised questions regarding the safety of bevacizumab in the peri-operative setting in patients undergoing liver resection due to potentially detrimental effects on the regenerative capacity of the liver. Preclinical studies indeed showed a slight impairment of liver regeneration by treatment with an anti-VEGFR2 antibody in a murine model of partial hepatectomy [44]. Interestingly, the results of the present study suggest the incidence of posthepatectomy liver failure of being less frequent in patients who receive chemotherapy together with bevacizumab. One must note that the above studies evaluated the role of VEGF and the effect of anti-angiogenic therapy on liver regeneration without concomitant use of cytotoxic chemotherapy. Furthermore, the decreased incidence of posthepatectomy liver failure after chemotherapy with bevacizumab may be mediated by protection from SOS and liver fibrosis.

Despite a beneficial effect of preoperative bevacizumab administration on parenchymal damage and functional recovery of the liver, we noted a significant increase in wound complications and total morbidity in case a high number of preoperative chemotherapy cycles was administered. Owing to these findings the positive effects of VEGF-targeted therapy on the hepatic parenchyma need to be weighed against potential risks. However, dissection of the molecular mechanisms responsible for decreased parenchymal damage might help to develop therapies specifically targeting pathways involved in chemotherapyassociated liver injury and in particular SOS.

Our study has several limitations. First, all studies included in the present meta-analysis were non-randomized studies and may therefore be affected by various sources of bias. Second, no uniform definitions as published before [45-47] were applied for the most relevant complications after hepatobiliary surgery. Third, we noted marked intra-and interstudy heterogeneity of the included studies with respect to the study designs, sample sizes, administered chemotherapy protocols and evaluated outcomes. We addressed these issues by robust methodology using a priori defined subgroup and sensitivity analyses together with a random effects model.

\section{Conclusion}

In conclusion, the results of the present systematic review and meta-analysis confirmed the safety of chemotherapy together with the anti-VEGF antibody bevacizumab in the 
perioperative treatment of patients with CLM. The protective effects from SOS and hepatic fibrosis warrant further investigation and may at least in part serve as an explanation for the unexpected finding that treatment with bevacizumab reduces the incidence of posthepatectomy liver failure.

\section{Additional files}

Additional file 1: Table S1. Histological analysis and parenchymal damage reported in studies on preoperative chemotherapy with and without bevacizumab (DOC $55 \mathrm{~kb}$ )

Additional file 2: Table S2. Definition of fibrosis in available studies (DOC $43 \mathrm{~kb}$ )

\section{Abbreviations \\ CLM: colorectal liver metastases; PDGFR: platelet derived growth factor receptor; SOS: sinusoidal obstruction syndrome; VEGF: vascular endothelial growth factor.}

\section{Competing interests}

The authors declare that they have no competing interests.

\section{Authors' contributions}

All authors have seen and approved the final version of the manuscript. The detailed contributions of each author are: AV: study design, data acquisition, data analysis, data interpretation, drafting of the manuscript. JF: study design, data acquisition, data analysis, data interpretation, manuscript revision. CR: data analysis, data interpretation, manuscript revision. GW: data acquisition, data analysis, manuscript revision. JW: study design, data interpretation, manuscript revision. NNR: study design, data acquisition, data analysis, data interpretation, drafting of the manuscript.

\section{Received: 21 June 2015 Accepted: 28 January 2016}

\section{(t)}

\section{References}

1. de Jong MC, Pulitano C, Ribero D, et al. Rates and patterns of recurrence following curative intent surgery for colorectal liver metastasis: an international multi-institutional analysis of 1669 patients. Ann Surg. 2009:250(3):440-8.

2. Nordlinger B, Sorbye $H$, Glimelius $B$, et al. Perioperative FOLFOX4 chemotherapy and surgery versus surgery alone for resectable liver metastases from colorectal cancer (EORTC 40983): long-term results of a randomised, controlled, phase 3 trial. Lancet Oncol. 2013;14(12):1208-15.

3. Giacchetti S, Perpoint B, Zidani R, et al. Phase III multicenter randomized trial of oxaliplatin added to chronomodulated fluorouracil-leucovorin as first-line treatment of metastatic colorectal cancer. J Clin Oncol. 2000;18(1):136-47.

4. Saltz LB, Cox JV, Blanke C, et al. Irinotecan plus fluorouracil and leucovorin for metastatic colorectal cancer. Irinotecan Study Group. N Engl J Med. 2000;343(13):905-14

5. Folprecht G, Gruenberger T, Bechstein WO, et al. Tumour response and secondary resectability of colorectal liver metastases following neoadjuvant chemotherapy with cetuximab: the CELIM randomised phase 2 trial. Lancet Oncol. 2010;11(1):38-47.

6. Hurwitz H, Fehrenbacher L, Novotny W, et al. Bevacizumab plus irinotecan, fluorouracil, and leucovorin for metastatic colorectal cancer. N Engl J Med. 2004;350(23):2335-42.

7. Mahfud M, Breitenstein S, El-Badry AM, et al. Impact of preoperative bevacizumab on complications after resection of colorectal liver metastases: case-matched control study. World J Surg. 2010;34(1):92-100.

8. Ribero D, Wang H, Donadon $\mathrm{M}$, et al. Bevacizumab improves pathologic response and protects against hepatic injury in patients treated with oxaliplatin-based chemotherapy for colorectal liver metastases. Cancer. 2007:110(12):2761-7

9. Rubbia-Brandt L. Sinusoidal obstruction syndrome. Clin Liver Dis. 2010;14(4):651-68.
10. Millet $\mathrm{G}$, Truant $\mathrm{S}$, Leteurtre $\mathrm{E}$, et al. Volumetric analysis of remnant liver regeneration after major hepatectomy in bevacizumab-treated patients: a case-matched study in 82 patients. Ann Surg. 2012;256(5):755-61. discussion 761-2.

11. Moher D, Liberati A, Tetzlaff J, et al. Preferred reporting items for systematic reviews and meta-analyses: the PRISMA statement. PLoS Med. 2009:6(7), e1000097.

12. Higgins JP, Green S. Cochrane Handbook for Systematic Reviews of Interventions Version 5.1.0 [updated March 2011]. The Cochrane Collaboration 2011. BMJ 2011;343:d5928

13. Atkins $D$, Best $D$, Briss PA, et al. Grading quality of evidence and strength of recommendations. BMJ. 2004:328(7454):1490.

14. Guyatt GH, Oxman AD, Vist GE, et al. GRADE: an emerging consensus on rating quality of evidence and strength of recommendations. BMJ. 2008;336(7650):924-6.

15. Rahbari NN, Aigner M, Thorlund K, et al. Meta-analysis shows that detection of circulating tumor cells indicates poor prognosis in patients with colorectal cancer. Gastroenterology. 2010;138(5):1714-26.

16. Rahbari NN, Bork U, Motschall E, et al. Molecular detection of tumor cells in regional lymph nodes is associated with disease recurrence and poor survival in node-negative colorectal cancer: a systematic review and metaanalysis. J Clin Oncol. 2012;30(1):60-70.

17. DerSimonian R, Laird N. Meta-analysis in clinical trials. Control Clin Trials. 1986;7(3):177-88.

18. Higgins JP, Thompson SG, Deeks JJ, et al. Measuring inconsistency in meta-analyses. BMJ. 2003:327(7414):557-60.

19. Egger M, Davey SG, Schneider M, et al. Bias in meta-analysis detected by a simple, graphical test. BMJ. 1997;315(7109):629-34.

20. Aussilhou B, Dokmak S, Faivre S, et al. Preoperative liver hypertrophy induced by portal flow occlusion before major hepatic resection for colorectal metastases can be impaired by bevacizumab. Ann Surg Oncol. 2009;16(6):1553-9.

21. Constantinidou A, Cunningham D, Shurmahi F, et al. Perioperative chemotherapy with or without bevacizumab in patients with metastatic colorectal cancer undergoing liver resection. Clin Colorectal Cancer. 2013;12(1):15-22.

22. D'Angelica M, Kornprat $P$, Gonen $M$, et al. Lack of evidence for increased operative morbidity after hepatectomy with perioperative use of bevacizumab: a matched case-control study. Ann Surg Oncol. 2007;14(2):759-65.

23. Kesmodel SB, Ellis LM, Lin E, et al. Preoperative bevacizumab does not significantly increase postoperative complication rates in patients undergoing hepatic surgery for colorectal cancer liver metastases. J Clin Oncol. 2008;26(32):5254-60.

24. Klinger M, Eipeldauer S, Hacker S, et al. Bevacizumab protects against sinusoidal obstruction syndrome and does not increase response rate in neoadjuvant XELOX/FOLFOX therapy of colorectal cancer liver metastases. Eur J Surg Oncol. 2009;35(5):515-20.

25. Lubezky N, Winograd E, Papoulas M, et al. Perioperative complications after neoadjuvant chemotherapy with and without bevacizumab for colorectal liver metastases. J Gastrointest Surg. 2013;17(3):527-32

26. Pessaux P, Panaro F, Casnedi S, et al. Targeted molecular therapies (cetuximab and bevacizumab) do not induce additional hepatotoxicity: preliminary results of a case-control study. Eur J Surg Oncol. 2010;36(6):575-82.

27. Reddy SK, Morse MA, Hurwitz $\mathrm{Hl}$, et al. Addition of bevacizumab to irinotecan- and oxaliplatin-based preoperative chemotherapy regimens does not increase morbidity after resection of colorectal liver metastases. J Am Coll Surg. 2008:206(1):96-106.

28. Rong Z, Martel G, Vandenbroucke-Menu F, et al. Impact of peri-operative bevacizumab on survival in patients with resected colorectal liver metastases: an analysis of the LiverMetSurvey. HPB (Oxford). 2014;16(4):342-9.

29. Rubbia-Brandt L, Lauwers GY, Wang H, et al. Sinusoidal obstruction syndrome and nodular regenerative hyperplasia are frequent oxaliplatin-associated liver lesions and partially prevented by bevacizumab in patients with hepatic colorectal metastasis. Histopathology. 2010;56(4):430-9.

30. Tamandl D, Gruenberger B, Klinger $M$, et al. Liver resection remains a safe procedure after neoadjuvant chemotherapy including bevacizumab: a case-controlled study. Ann Surg. 2010;252(1):124-30.

31. van der Pool AE, Marsman HA, Verheij J, et al. Effect of bevacizumab added preoperatively to oxaliplatin on liver injury and complications after resection of colorectal liver metastases. J Surg Oncol. 2012:106(7):892-7.

32. Vera R, Gomez Dorronsoro M, Lopez-Ben S, et al. Retrospective analysis of pathological response in colorectal cancer liver metastases following treatment with bevacizumab. Clin Trans/ Oncol 2014;16(8):739-45 
33. Wicherts DA, de Haas RJ, Sebagh M, et al. Impact of bevacizumab on functional recovery and histology of the liver after resection of colorectal metastases. Br J Surg. 2011;98(3):399-407.

34. Zorzi D, Chun YS, Madoff DC, et al. Chemotherapy with bevacizumab does not affect liver regeneration after portal vein embolization in the treatment of colorectal liver metastases. Ann Surg Oncol. 2008;15(10):2765-72.

35. Rubbia-Brandt L, Audard V, Sartoretti P, et al. Severe hepatic sinusoidal obstruction associated with oxaliplatin-based chemotherapy in patients with metastatic colorectal cancer. Ann Oncol. 2004;15(3):460-6.

36. Rubbia-Brandt L, Tauzin S, Brezault C, et al. Gene expression profiling provides insights into pathways of oxaliplatin-related sinusoidal obstruction syndrome in humans. Mol Cancer Ther. 2011;10(4):687-96.

37. Robinson SM, Mann J, Vasilaki A, et al. Pathogenesis of FOLFOX induced sinusoidal obstruction syndrome in a murine chemotherapy model. J Hepatol. 2013:59(2):318-26.

38. Wang $Y$, Gao J, Zhang $D$, et al. New insights into the antifibrotic effects of sorafenib on hepatic stellate cells and liver fibrosis. J Hepatol. 2010;53(1):132-44.

39. Chen $Y$, Huang $Y$, Reiberger $T$, et al. Differential effects of sorafenib on liver versus tumor fibrosis mediated by stromal-derived factor 1 alpha/C-X-C receptor type 4 axis and myeloid differentiation antigen-positive myeloid cell infiltration in mice. Hepatology. 2014:59(4):1435-47.

40. Adnane L, Trail PA, Taylor I, et al. Sorafenib (BAY 43-9006, Nexavar), a dual-action inhibitor that targets RAF/MEK/ERK pathway in tumor cells and tyrosine kinases VEGFR/PDGFR in tumor vasculature. Methods Enzymol. 2006:407:597-612

41. Pinzani M, Milani S, Herbst $H$, et al. Expression of platelet-derived growth factor and its receptors in normal human liver and during active hepatic fibrogenesis. Am J Pathol. 1996;148(3):785-800.

42. Michalopoulos GK, DeFrances MC. Liver regeneration. Science. 1997:276(5309):60-6.

43. Redaelli CA, Semela D, Carrick FE, et al. Effect of vascular endothelial growth factor on functional recovery after hepatectomy in lean and obese mice. J Hepatol. 2004;40(2):305-12

44. Van Buren G, Yang 2nd AD, Dallas NA, et al. Effect of molecular therapeutics on liver regeneration in a murine model. J Clin Oncol. 2008;26(11):1836-42.

45. Koch M, Garden OJ, Padbury R, et al. Bile leakage after hepatobiliary and pancreatic surgery: a definition and grading of severity by the International Study Group of Liver Surgery. Surgery. 2011;149(5):680-8.

46. Rahbari NN, Garden OJ, Padbury R, et al. Posthepatectomy liver failure: a definition and grading by the International Study Group of Liver Surgery (ISGLS). Surgery. 2011;149(5):713-24.

47. Rahbari NN, Garden OJ, Padbury R, et al. Post-hepatectomy haemorrhage: a definition and grading by the International Study Group of Liver Surgery (ISGLS). HPB (Oxford). 2011;13(8):528-35.

\section{Submit your next manuscript to BioMed Central and we will help you at every step:}

- We accept pre-submission inquiries

- Our selector tool helps you to find the most relevant journal

- We provide round the clock customer support

- Convenient online submission

- Thorough peer review

- Inclusion in PubMed and all major indexing services

- Maximum visibility for your research

Submit your manuscript at www biomedcentral.com/submit

) Biomed Central 